\title{
Construction of a CXCL12-KDEL Fusion Gene to Inhibit Head and Neck Squamous Cell Carcinoma Metastasis by Intracellular Sequestration of CXCR4
}

\author{
Wenchao Zhang, Xudong Wang, Kai Yue, Su Liu, and Xiaonan Liu \\ Department of Maxillofacial and ENT, National Cancer Clinical Research Center, \\ Tianjin Medical University Cancer Institute \& Hospital, Tianjin 300060, China
}

Correspondence should be addressed to Xudong Wang; zwbeyond_999@sina.com

Received 26 June 2014; Revised 18 August 2014; Accepted 19 August 2014

Academic Editor: Shiwu Zhang

Copyright (C) 2015 Wenchao Zhang et al. This is an open access article distributed under the Creative Commons Attribution License, which permits unrestricted use, distribution, and reproduction in any medium, provided the original work is properly cited.

\begin{abstract}
The CXCL12-CXCR4 biological axis consisting of the chemotactic factor CXCL12 and its specific receptor CXCR4 plays an important role in oral cancer metastasis. High expression of CXCR4 may help oral squamous cancer cells invade local tissues and metastasize to lymph nodes. No obvious association was observed between CXCL12 expression and lymph node metastasis, suggesting that CXCL12 chemotaxis may only be related to CXCR4 expression on the tumor cell membrane. KDEL can be retained by receptors on the surface of the intracellular endoplasmic reticulum (ER) and also be called an ER retention signal sequence. So we adopted the KDEL sequence in this study to generate a CXCL12-KDEL fusion protein in combination with a traceable E-tag label. As such, CXCL12 was retained in the ER. Specific receptor CXCR4 binds to the CXCL12-KDEL, was also retained in the ER, and was thus prevented from reaching the oral squamous cancer cell surface. We reduced the cell surface level of CXCR4 and called the technique "intracellular sequestration." By this way, we have finished blocking of CXCL12-CXCR4 biological axis and inhibiting lymph node metastasis of oral carcinoma.
\end{abstract}

\section{Introduction}

Head and neck squamous cell carcinoma (HNSCC) is the most common malignant tumor of the oral cavity and throat, and subsequent neck lymph node metastases have important influence on prognosis [1-4]. The CXCL12-CXCR4 biological axis consisting of the chemotactic factor CXCL12 and its specific receptor CXCR4 plays an important role in cancer metastasis [5-7]. This axis facilitates tumor metastasis in breast cancer, non-small cell lung cancer, rhabdomyosarcoma, and other human malignant tumors, and the blocking of CXCL12-CXCR4 biological axis inhibits metastasis [8-11].

KDEL signal sequence is located in the carboxyl end of structural and functional proteins in the endoplasmic reticulum (ER). It represents a four-peptide sequence: LysAsp-Glu-Leu. Relevant receptors for the sequence in the Golgi membrane can recognize KDEL signals and combine with them, and then the combined ER proteins will be carried back to ER. KDEL can be retained by receptors on the surface of the intracellular ER and also be called an endoplasmic reticulum (ER) retention signal sequence.

Here we have made use of "intracellular sequestration" to reduce the cell surface level of CXCR4 by constructing CXCL12-KDEL fusion gene. Specific receptor CXCR4 binds to the CXCL12-KDEL, is also retained in the ER, and is thus prevented from reaching the $\mathrm{Tb}$ squamous cancer cell surface.

We aim to analyze the role of the CXCL12-CXCR4 biological axis on HNSCC lymph node metastasis. This will be achieved by constructing and utilizing a CXCL12-KDEL fusion gene expression vector (CXCL12-KDEL-pIRES2EGFP) to block the CXCL12-CXCR4 biological axis and intracellularly sequester CXCR4, in order to inhibit HNSCC metastasis.

\section{Materials and Methods}

2.1. General Data. All the samples were collected from patients admitted to the Tianjin Medical University Cancer 
Hospital between January 2005 and December 2006. Tissue samples surgically removed from 65 patients with HNSCC and 15 patients with benign lesions were included in this study, as the experimental and control groups, respectively. There were 43 men and 22 women, with an average age of $61 \mathrm{y}$ (range: 19 to $83 \mathrm{y}$ ). Patients were staged according to the TNM staging criteria (2012) designed by the Union for International Cancer Control (UICC). In all, there were 26 cases in stages I-II and 39 cases in stages III-IV. Among the 65 patients of squamous cell carcinomas, 35 patients had ipsilateral and/or contralateral neck lymph node metastases, 30 patients had no lymph node metastases, and 2 patients had distant metastases. None of the patients received preoperative chemotherapy and radiotherapy. The carcinoma diagnosis was histopathologically confirmed with complete clinical and pathological data.

2.2. Experimental Materials. Competent Escherichia coli cells JM109, pMD19T plasmid, and DH5 $\alpha$ cell were purchased from Takara Shuzo Co., Ltd. (Kyoto, Japan) and Promega (Madison, WI, USA), respectively. PIRES2-EGFP plasmid was prepared in our laboratory. Superscript II reverse transcription kit and PCR products extraction kit were purchased from Qiagen (Hilden, Germany) and Invitrogen Corporation (Maryland, USA), respectively. PCR purification and DNA connection kits were purchased from Takara Shuzo Co., Ltd. (Kyoto, Japan) and Roche Company (USA), respectively. RPMI-1640 culture medium was purchased from Invitrogen Company (USA) and fetal bovine serum (FBS) was purchased from Gibco Company (USA). Human tongue squamous cancer cell line $\mathrm{Tb}$ was provided by Shanghai Jiaotong University affiliated Ninth People's Hospital. Goat polyclonal antibody against CXCL12 was purchased from Santa Cruz Company (USA); horseradish peroxidase-labeled second antibody and mouse anti- $\beta$-actin antibody polyclonal antibody were purchased from Beijing Golden Bridge Biotechnology Company. RIPA lysis buffer was purchased from Millipore Company (USA).

\subsection{Experimental Methods}

\subsubsection{Expression and Localization of CXCL12 and CXCR4 in Primary Tumors and Metastatic Lymph Nodes}

(1) Expression and Localization of CXCL12 and CXCR4 in HNSCC and Lymph Nodes by Immunohistochemistry. CXCR4 and CXCL12 were detected using a rabbit anti-human polyclonal antibody (Boster Company, Wuhan, China) and rabbit anti-human monoclonal antibody (Santa Cruz, USA), respectively. Experiments were performed according to the manufacturer's instructions.

(2) CXCR4 mRNA Levels in HNSCC Metastasis Group, HNSCC Nonmetastasis Group, and Control Group and CXCL12 mRNA Levels in Metastatic Lymph Nodes and Nonmetastasis Group by RT-PCR. Total RNA was extracted using Trizol (Invitrogen, Carlsbad, CA, USA), following the manufacturer's protocol. RT reactions were performed in a final volume of $20 \mu \mathrm{L}$ using M-MLT reverse transcriptase, according to the manufacturer instructions. The resulting cDNA products were stored at $-20^{\circ} \mathrm{C}$. The primers used in this study were as follows: endogenous control $\beta$-actin: $5^{\prime}$-CCTGGGCATGGAGTCCTGTG-3' (forward), $5^{\prime}$-AGGGGCCGGACTCGTCATAC-3' (reverse); CXCLl2: $5^{\prime}$-GCCATGAACGCCAAGGTC-3' (forward), 5' -CGAGTGGGTCTAGCGGAAAG-3' (reverse), 312 bp; CXCR4; 5'-AGCTGTTGGCTGAAAAGGTGGTCTATG-3' (forward), $5^{\prime}$ GCGCTTCTGGTGGCCCTTGGAGTGTG-3' (reverse), 254 bp. PCR amplification of CXCR4 was performed under the following conditions: $94^{\circ} \mathrm{C}$ for $5 \mathrm{~min}$ followed by 30 cycles of $94^{\circ} \mathrm{C}$ for $1 \mathrm{~min}, 56^{\circ} \mathrm{C}$ for $1 \mathrm{~min}$, and $72^{\circ} \mathrm{C}$ for $1 \mathrm{~min}$, with a final extension at $72^{\circ} \mathrm{C}$ for $10 \mathrm{~min}$. PCR amplification of CXCL12 was performed under the following conditions: $94^{\circ} \mathrm{C}$ for $5 \mathrm{~min}$, followed by 30 cycles of $94^{\circ} \mathrm{C}$ for $1 \mathrm{~min}, 55^{\circ} \mathrm{C}$ for $1 \mathrm{~min}$, and $72^{\circ} \mathrm{C}$ for $1 \mathrm{~min}$, with a final extension at $72^{\circ} \mathrm{C}$ for $10 \mathrm{~min}$. RT-PCR products were detected by agarose gel electrophoresis.

\subsubsection{Construction of the CXCL12-KDEL Fusion Gene}

(1) CXCL12-KDEL Fusion Gene Primer Design and Fragments Amplification. CXCL12 coding gene sequences were retrieved from GenBank to determine the full amplification sequence. Once this was determined, cellular RNA was used as template, and the CXCL12-KDEL fragment was amplified by RT$\mathrm{PCR}$, resulting in an amplified fragment of $350 \mathrm{bp}$. The CXCL12-KDEL fusion gene primers used were as follows: $5^{\prime}$ TAGCAGATCTGCCATGGACGCCAAG-3' (forward) and 5' -TAGCGTCGACTTACAGCTCGTCCTTCTCGCTTCGCGGTTCCAGCGGATCCGGATACGGCACCGGCGCACCCTTGTTTAAAGCTTTCTCCAGGTA-3' (reverse); they were synthesized by SBS Genetech Co., Ltd. (Beijing, China). The ER retention sequence KDEL and the fusion gene's detecting marker genes sequence (E-tag) were added to reverse primers. The final PCR amplification reaction consisted of the following components: $5 \mu \mathrm{L}$ of $10 \mathrm{x}$ PCR buffer, $4 \mu \mathrm{L}$ of $4 \mathrm{x}$ dNTP $\left(2.5 \mathrm{mM}\right.$, each), $3 \mu \mathrm{L}$ of $\mathrm{MgCl}_{2}$ $(25 \mathrm{mM}), 1 \mu \mathrm{L}$ each of $\mathrm{P} 1$ and $\mathrm{P} 2$ primers (both at $200 \mathrm{pmol})$, $1 \mu \mathrm{L}$ of RNA template, $0.5 \mu \mathrm{L}$ of Taq polymerase $(2.5 \mathrm{U} / \mu \mathrm{L})$, and $34.5 \mu \mathrm{L}$ of double distilled water. The PCR reaction conditions were as follows: $94^{\circ} \mathrm{C}$ for $5 \mathrm{~min}$, followed by 30 cycles of $94^{\circ} \mathrm{C}$ for $1 \mathrm{~min}, 62^{\circ} \mathrm{C}$ for $1 \mathrm{~min}$, and $72^{\circ} \mathrm{C}$ for $1 \mathrm{~min}$. The resulting 350-bp PCR product was separated and analyzed by electrophoresis on a $1 \%$ agarose gel, followed by purification and recovery.

(2) Amplification and Identification of Recombinant pMD19$T$ Vector. The purified CXCL12-KDEL gene was inserted into the pMD19-T vector to obtain a recombinant vector that was subsequently amplified and sequence verified.

(3) Construction of CXCL12-KDEL-pIRES2-EGFP Plasmid Eukaryotic Expression Vector. The amplified products were then subcloned into a pIRES2-EGFP plasmid, transformed into DH $5 \alpha$ competent cells, and cultured overnight on a Luria broth agar plate containing kanamycin, in a $37^{\circ} \mathrm{C}$ constant temperature incubator. Single colonies were picked from the plate, following which plasmid DNA was extracted as per the 
manufacturer's protocol. The resulting DNA was subjected to restriction digestion using two enzymes, BglII and SalI, and $5 \mu \mathrm{L}$ of the digested sample was separated by agarose gel electrophoresis. Positive clones identified by restriction enzyme digestion were then sequenced by Takara Company (Dalian, China). The resulting recombinant construct is referred to as CXCL12-KDEL-pIRES2-EGFP.

\subsubsection{Recombinant Plasmid Transfection}

(1) Tb Cell Culture and Gene Transfection. Tb cells were cultured in RPMI 1640 medium and supplemented with $10 \%$ FBS, $4 \mathrm{mM}$ L-glutamine, $50 \mu / \mathrm{mL}$ penicillin, and $50 \mu \mathrm{g} / \mathrm{mL}$ streptomycin (Invitrogen, Carlsbad, CA, USA) at $37^{\circ} \mathrm{C}$ under $5 \% \mathrm{CO}_{2}$. Tb cells in logarithmic growth phase were seeded in 6-well plates, with $3 \times 10^{5}$ cells in each well. When the cells were $80 \%$ confluent, growth medium was removed, the cells were washed twice with PBS, and $2 \mathrm{~mL}$ of RPMI1640 medium without serum was added back, and the cells were placed in an incubator at $37^{\circ} \mathrm{C}$ for $40 \mathrm{~min}$. CXCL12KDEL-pIRES2-EGFP plasmid $(5 \mu \mathrm{L})$ was combined with Lipofectamine $(10 \mu \mathrm{L})$ and added to the cell culture medium. The cells were then incubated at $37^{\circ} \mathrm{C}$ for $6 \mathrm{~h}$, following which the medium was replaced with fresh complete medium and the cells were incubated for an additional $48 \mathrm{~h}$ until harvest.

(2) Assay for Transfection Efficiency. Tb3.1 cells cultured for 48-72 h after transfection with CXCL12-KDEL-pIRES2EGFP plasmid were evaluated for transfection efficiency by measuring levels of fluorescent protein expression using a DP70 fluorescence inverted phase contrast microscope. Fluorescent and bright field images were analyzed using the IPP5.1 software (Olympus Company, Japan).

\subsubsection{Recombinant Fusion Gene Functional Experiment}

(1) Determination of CXCL12-KDEL Protein Level by Western Blot. CXCL12-KDEL protein levels at $72 \mathrm{~h}$ after transfection were evaluated by measuring levels of the E-tag label using western blot. Cells were divided into six groups: CXCL12KDEL-pIRES2-EGFP transfection group, empty vector pIRES2-EGFP transfection group, nontransfection group, and culture supernatants from each of the three groups.

(2) Analysis of Surface CXCR4 Expression in Transfected Cells. Transfected cells were harvested and incubated with CXCR4 antibody, following which they were analyzed by flow cytometry. Cells transfected with CXCL12-KDEL-pIRES2EGFP were compared to cells transfected with the empty vector pIRES2-EGFP.

(3) Cell Chemotaxis Assay. For the chemotaxis assay, cancer cells were added to the upper layer of a chemotaxis chamber and recombinant CXCL12 was added to the bottom layer. Cells were counted and analyzed after incubation at $37^{\circ} \mathrm{C}$ for $2 \mathrm{~h}$. Cells were divided into three groups: CXCL12-KDELpIRES2-EGFP transfection group, empty vector pIRES2EGFP transfection group, and nontransfection group.

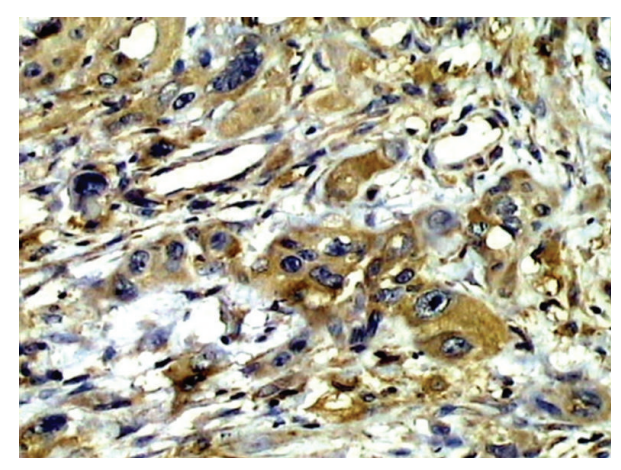

FIGURE 1: Strong positive expression of CXCR4 in metastatic squamous carcinoma tissues (200x).

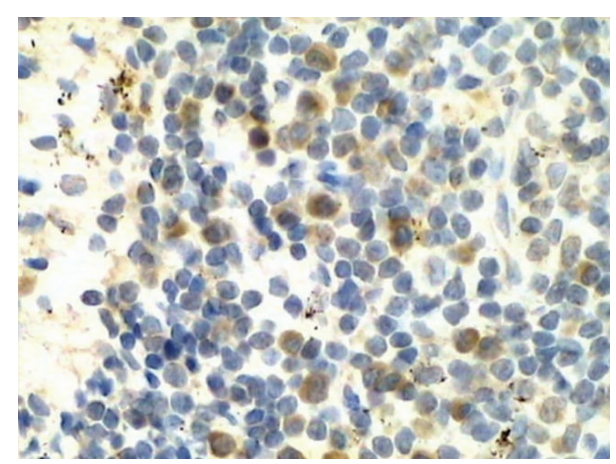

FIgURe 2: Positive expression of CXCL12 in lymph node tissues (200x).

2.4. Statistical Analysis. All the statistical analyses were performed using SPSS 16.0 (SPSS, Chicago, IL, USA). IHC results were estimated using $\chi^{2}$ test or Fisher's exact test; RT-PCR results were estimated using ANOVA and two-sample $t$-test. A $P$ value of $<0.05$ indicates a significant difference.

\section{Results}

3.1. Immunohistochemical Results. 65 patient's specimens were tested by IHC. CXCR4 was mostly expressed in squamous carcinoma tissues and localized mainly to the cytoplasm and partially to the cell membrane (Figure 1). CXCL12 was expressed in lymph node tissues, primarily in lymphocytes, and localized to the intercellular compartments (Figure 2).

Statistical analysis showed that the positive expression of CXCR4 in stages III-IV was significantly higher than that in stages I-II group $(P=0.00)$. Similarly, it was higher in G3 group than that in G1-G2 group $(P=0.00)$ and higher in metastatic group than that in the nonmetastatic group $(P=$ 0.017) (Table 1).

There were no significant differences of CXCL12 positive expression in all compared groups $(P>0.05)$ (Table 1$)$.

3.2. RT-PCR. Statistical analysis of integral optical density (IOD) showed that CXCR4 expression in metastatic group was higher than that in the nonmetastatic group and control 
TABLE 1: The expression of CXCL12/CXCR4 by IHC in different groups of patients.

\begin{tabular}{|c|c|c|c|c|c|c|c|}
\hline \multirow{2}{*}{ Clinicopathological parameter } & \multirow{2}{*}{$n$} & \multicolumn{2}{|c|}{ Positive (\%) } & \multicolumn{2}{|c|}{$\chi^{2}$} & \multicolumn{2}{|c|}{$P$ value } \\
\hline & & CXCR4 & CXCL12 & CXCR4 & CXCL12 & CXCR4 & CXCL12 \\
\hline Gender & & & & 2.694 & 0.756 & 0.101 & 0.384 \\
\hline Male & 43 & $23(53.5)$ & $12(27.9)$ & & & & \\
\hline Female & 22 & $12(54.5)$ & $6(27.3)$ & & & & \\
\hline Age & & & & 1.234 & 0.618 & 0.267 & 0.432 \\
\hline$<60$ ys & 31 & $16(51.6)$ & $8(25.8)$ & & & & \\
\hline$\geq 60 \mathrm{ys}$ & 34 & $19(55.9)$ & $10(29.4)$ & & & & \\
\hline Stage & & & & 16.44 & 0.016 & 0.000 & 0.900 \\
\hline I-II & 26 & $7(26.9)$ & $7(26.9)$ & & & & \\
\hline III-IV & 39 & $28(71.8)$ & $11(28.2)$ & & & & \\
\hline Differentiation & & & & 27.41 & 1.025 & 0.000 & 0.311 \\
\hline G1-G2 & 47 & $20(42.6)$ & $13(27.7)$ & & & & \\
\hline G3 & 18 & $15(83.3)$ & $5(27.8)$ & & & & \\
\hline Metastasis of lymph node & & & & 5.704 & 0.296 & 0.017 & 0.587 \\
\hline No & 30 & $10(33.3)$ & $8(26.7)$ & & & & \\
\hline Yes & 35 & $25(71.4)$ & $10(28.6)$ & & & & \\
\hline
\end{tabular}

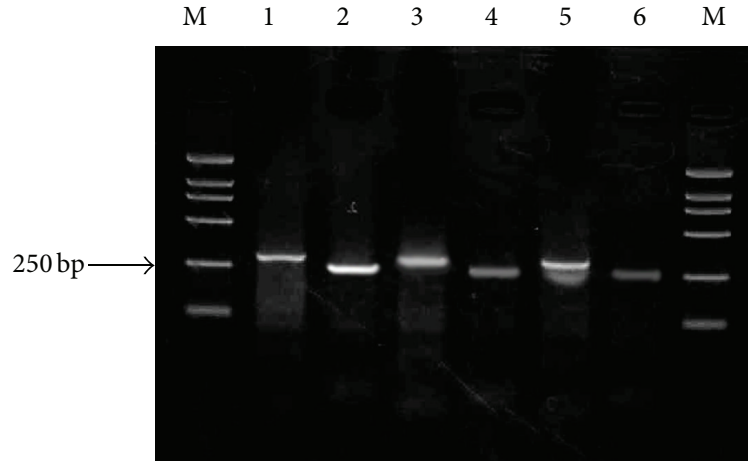

M: DL2000;1 ,3 , and 5: $\beta$-actin (305bp); 2: CXCR4(254bp)

expression in HNSCC with lymph node metastasis;

4: CXCR4 expression in nonmetastatic HNSCC; 6: CXCR4

expression in benign tumor control group

FIGURE 3: CXCR4 expression in primary head and neck squamous carcinoma (HNSCC) tissues.

TABLE 2: RT-PCR results of CXCR4 expression level in oral squamous cell carcinomas.

\begin{tabular}{lcc}
\hline Group & $n$ & CXCR4 IOD \\
\hline Normal oral tissues and benign lesions & 15 & $0.406 \pm 0.044^{* * *}$ \\
Nonmetastatic SCC & 15 & $0.464 \pm 0.068^{*}$ \\
Lymph node metastatic SCC & 15 & $0.900 \pm 0.108^{* *}$ \\
\hline
\end{tabular}

SCC: squamous cell carcinomas and ${ }^{*, * *} P<0.05$.

${ }^{* *} \mathrm{VS}^{*} P<0.05,{ }^{* *} \mathrm{VS}^{* * *} P<0.05,{ }^{*} \mathrm{VS}^{* * *} P<0.05$.

group $(P<0.05)$; CXCR4 expression in the nonmetastatic group was significantly higher than that in the positive control group $(P<0.05)$ (as shown in Figure 3 and Table 2$)$.

Statistical analysis showed that the expression of CXCR4 in lymph nodes with metastatic tumor was significantly

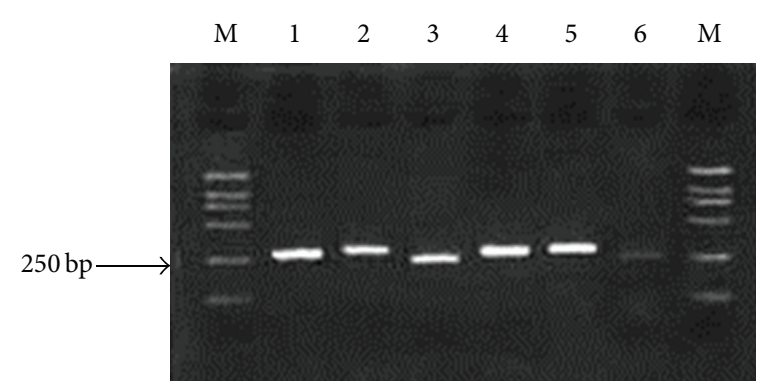

M: DL2000; 1, 4: $\beta$-actin (305bp); 2: CXCL12 (312 bp)

expression in metastatic lymph node; 3: CXCR4 (254 bp)

expression in metastatic lymph node; 5: CXCL12 expression

in nonmetastatic lymph node; 6: CXCR4 expression in

nonmetastatic lymph node

Figure 4: CXCL12 and CXCR4 expression levels in neck lymph nodes.

higher than that of the nonmetastatic lymph nodes $(P<0.05)$, and there was no significant difference of CXCL12 expression in lymph nodes between the two groups $(P>0.05)$ (as shown in Figure 4 and Table 3$).$

3.3. Amplification of CXCL12-KDEL Gene. The expected and observed size of the amplified fragment were $350 \mathrm{bp}$ (Figure 5).

3.4. Verification of Recombinant Plasmid by Enzyme Digestion and Sequencing. A BglII/SalI restriction digest of CXCL12KDEL-pIRES2-EGFP plasmid yields two predicted fragments of $350 \mathrm{bp}$ and $5.3 \mathrm{~kb}$. The length of the inserted product was confirmed to be of the same size as the predicted enzyme 
TABLE 3: RT-PCR results of CXCL12 and CXCR4 expression level in neck lymph nodes (IOD).

\begin{tabular}{llcr}
\hline Group & $n$ & CXCL12 $^{*}$ & CXCR4 $^{* *}$ \\
\hline Metastatic lymph nodes & 7 & $0.935 \pm 0.087$ & $0.947 \pm 0.042$ \\
Nonmetastatic lymph nodes & 7 & $0.861 \pm 0.047$ & $0.396 \pm 0.071$ \\
\hline
\end{tabular}

${ }^{*} P>0.05,{ }^{* *} P<0.05$.

TABLE 4: Chemotactic cells count and chemotactic index in each group $(\bar{x} \pm s, n=3)$.

\begin{tabular}{lcc}
\hline Group & Cell count & CI value \\
\hline Nontransfection group & $825.67 \pm 62.80$ & 1 \\
Empty vector (pIRES2-EGFP) transfection group & $711.33 \pm 49.54$ & 0.86 \\
CXCL12-KDEL transfection group & $216.00 \pm 84.12$ & 0.26 \\
\hline
\end{tabular}

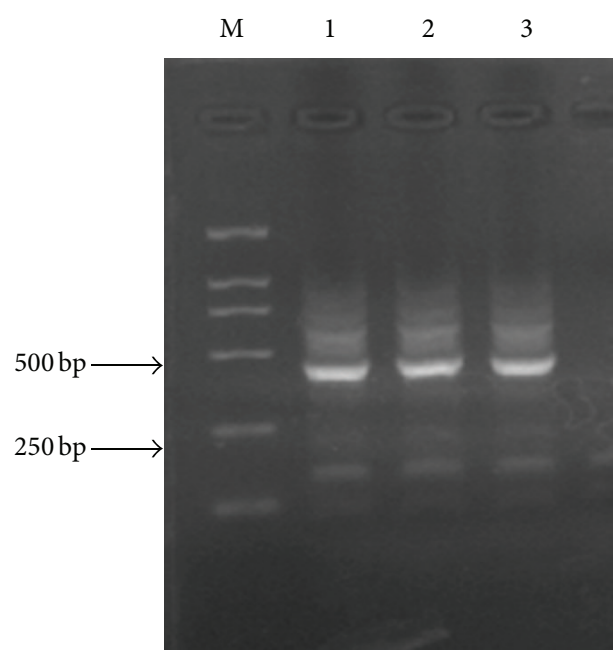

FIGURE 5: Agarose gel electrophoretogram of CXCL12-KDEL fusion gene PCR products. M: DL2000; 1-3: CXCL12-KDEL (350 bp).

digestion product (Figure 6). The CXCL12-KDEL-pIRES2EGFP plasmid was also sequence verified to be identical to the known gene sequence.

3.5. Determination of CXCL12-KDEL-pIRES2-EGFP Plasmid Transfection Efficiency. We observed that $48 \mathrm{~h}$ after recombinant plasmid transfection, $45 \%$ of the cells were positive for the expression of green fluorescent protein in $\mathrm{Tb}$ cells, and at $72 \mathrm{~h}$ after transfection this had increased to $50 \%$ (Figure 7 ).

3.6. Determination of CXCL12 Protein Levels by Western Blot. IOD was collected for each sample after transfection with recombinant CXCL12-KDEL-pIRES2-EGFP. Tb cells showed expression of E-tag protein, which is a surrogate label for CXCL12, whereas no protein expression was detected in the cells and the culture supernatant of the cells transfected with an empty plasmid or of those left untransfected (Figure 8).

3.7. CXCR4 Cell Surface Expression. Compared with the control group, CXCR4 expression on the cell surface in the experimental group is clearly reduced after transfection (Figure 9).

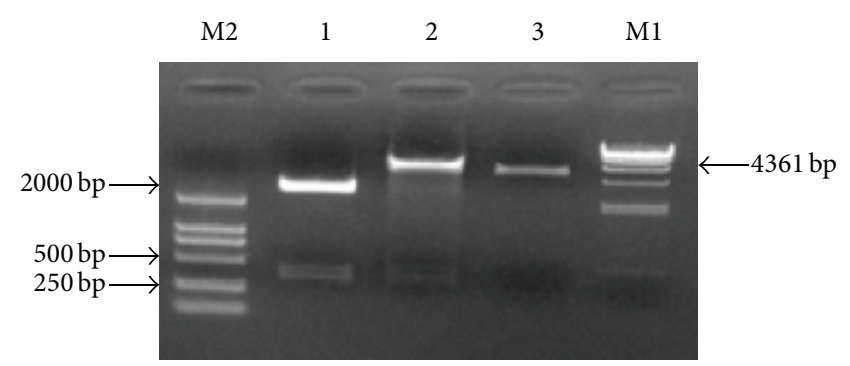

M1: $\lambda$-HindIII DNA Marker; 1: CXCL12-KDEL-19pMD-BglII/SalI; 2 :

CXCL12-KDEL-pIRES2-EGFP-BglII/SalI; 3: pIRES2-EGFP-BglII/SalI;

M2: DL 2, 000 DNA marker In the agarose gel electrophoretogram,

CXCL12-KDEL gene bands (350 bp), and 19 pMD vector bands

$(2.7 \mathrm{~kb})$ can be seen in Lane 1, CXCL12-KDEL gene bands ( $350 \mathrm{bp}$ ) and pIRES2-EGFP-vector bands ( $5.3 \mathrm{~kb}$ ) can be seen in Lane 2, and pIRES2-EGFP-vector bands ( $5.3 \mathrm{~kb}$ ) can be seen in Lane 3

FIgURE 6: Recombinant 19 pMD and pIRES2-EGFP-vector double digest gel electrophoretogram.

3.8. Chemotaxis Assay. Chemotaxis of cells in the experimental group was significantly less than that in the nontransfection group and the control group (transfected with empty vector $)(F=70.14, P=0.00)$. However, there was no statistically significant difference between the nontransfection group and the empty vector transfection group (Table 4).

\section{Discussion}

Chemokines are a family of small proinflammatory chemoattractant cytokines that bind to leukocyte-expressed seventransmembrane domain receptors and play a critical role in tumor angiogenesis and metastasis [12]. The chemokine CXCL12, also termed stromal cell derived factor-1 (SDF-1), is a member of the CXC chemokine family, of which CXCR4 is a known CXCL12-specific receptor $[13,14]$. CXCL12 is a small $(8 \mathrm{kDa})$ chemokine that was originally regarded as an efficacious lymphocyte chemoattractant and was characterized as a modulator of several physiological processes. [15-18]. CXCR4 plays a key role in tumor cell dissemination and metastasis development in the majority of cancers and several types of leukemia [19-23]. 

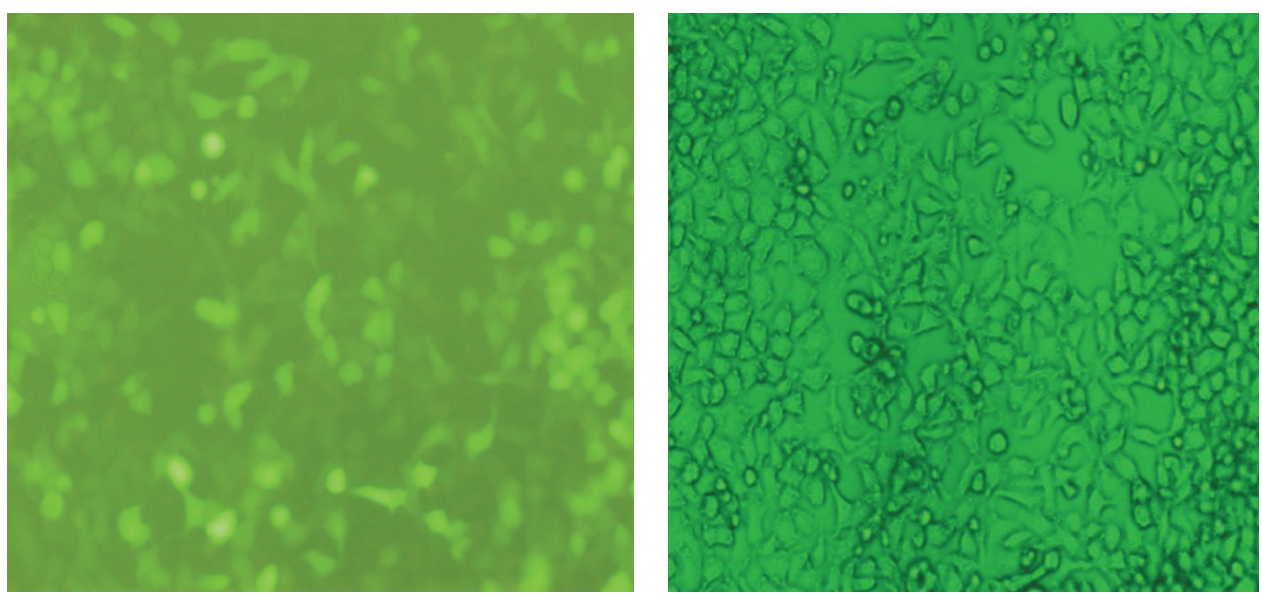

FIGURE 7: Analysis of Tb cells $72 \mathrm{~h}$ after transfection of recombinant CXCL12-KDEL-pIRES2-EGFP plasmid.

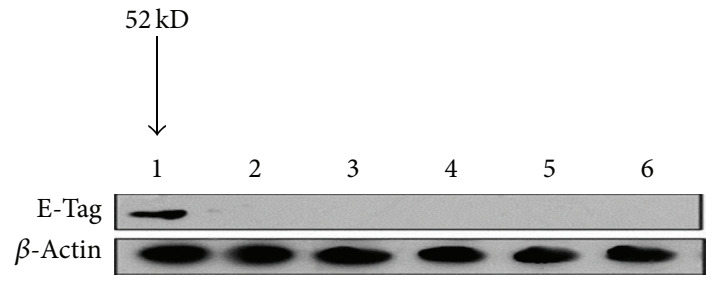

Figure 8: Western blot electrophoretogram of E-tag protein. 1: Etag protein $(52 \mathrm{kd})$ expression can be seen in Tb cells in the E-tag labeled CXCL12-KDEL transfection group, while no E-tag protein expression was observed in the remaining 5 groups; 2 : red vector pIRES2 EGFP transfection group; 3: normal Tb nontransfection cells; 4-6: the culture supernatant of three kinds of cells.

Thus, the CXCL12-CXCR4 biological axis formed by CXCL12 and its specific receptor CXCR4 plays an important role in the process of metastasis of cancer cells [24]. The biological axis is related to the invasion, recurrence, and metastasis in many types of cancer including breast cancer [25], non-small cell lung cancer (NSCL) [26], colon cancer $[27,28]$, oral cavity squamous carcinoma [9], esophageal cancer [29], pancreatic carcinoma [30], renal cell carcinoma [31], and endometrial cancer [32].

In this study, our results show high CXCR4 expression in HNSCC, with higher expression in the metastatic group compared to the nonmetastatic group, which indicates that CXCR4 may play an important role in HNSCC metastasis. No obvious association was observed between CXCL12 expression and lymph node metastasis, suggesting that CXCL12 chemotaxis may only be related to CXCR4 expression on the tumor cell membrane. We speculate that tumor cells with high expression of CXCR4 have strong potential for local invasion, and CXCL12 expressed in lymph nodes has a chemotactic effect on their directional migration.

In vitro experiments confirmed that blocking the CXCL12-CXCR4 signal axis activity by different molecular biology methods such as RNAi, small molecule inhibitors in breast cancer cells, could effectively reverse the malignant phenotype of the tumor cells [33-35]. Animal experiments also show that CXCR4 monoclonal antibody can inhibit lymph node metastasis of human breast cancer cells in nude mice. The ER retention signal sequence KDEL represents a four-peptide sequence (Lys-Asp-Glu-Leu), which can be retained by receptors on the surface of the ER [36]. Based on this, we adopted the KDEL sequence in this study to generate a CXCL2-KDEL fusion protein in combination with a traceable E-tag label. As such, CXCL12 was retained in the endoplasmic reticulum allowing it to act as a sink for CXCR4, thus sequestering the latter within the intracellular space. The intracellular sequestration of CXCR4 provides a new strategy for blocking the CXCL12-CXCR4 biological axis. The validity of the CXCL12-KDEL-pIRES2-EGFP plasmid was confirmed by both restriction enzyme digestion and sequencing. At 48 and $72 \mathrm{~h}$ after $\mathrm{Tb}$ cells were transfected with the recombinant CXCL12-KDEL-pIRES2-EGFP plasmid, we were able to observe fluorescent gene expression from EGFP, confirming successful transfection. Vectors can be used to express EGFP protein alone or obtain stable transfection cell lines. The transfection efficiency can then be analyzed by the intensity of fluorescent protein expression [37]. As determined by western blot of the E-tag label protein, a surrogate marker for CXCL12 protein was successfully expressed following recombinant plasmid transfection, whereas CXCL12 protein expression was not detected in cells transfected with the empty plasmid or in those that were left untransfected.

In this study, we adopted a technique that used CXCL12KDEL as an intrinsic factor for the intracellular sequestration of CXCR4. We inserted the KDEL sequence downstream of CXCL12 and through functional experiments were able to demonstrate the intracellular capture of CXCR4 surface expression and the blocking of the chemotaxis of cells via the CXCL12-CXCR4 biological axis [38]. This strategy of sequestering CXCR4 using another intracellular component is an example of technology using inactivated biomolecular molecules; other examples are the use of antisense nucleic acid, ribozymes-mediated negative mutation, and gene knockout technologies. These technologies are very similar to the "intracellular antibody" technology, ultimately aimed to form a phenotypic knockout model. In this study, 

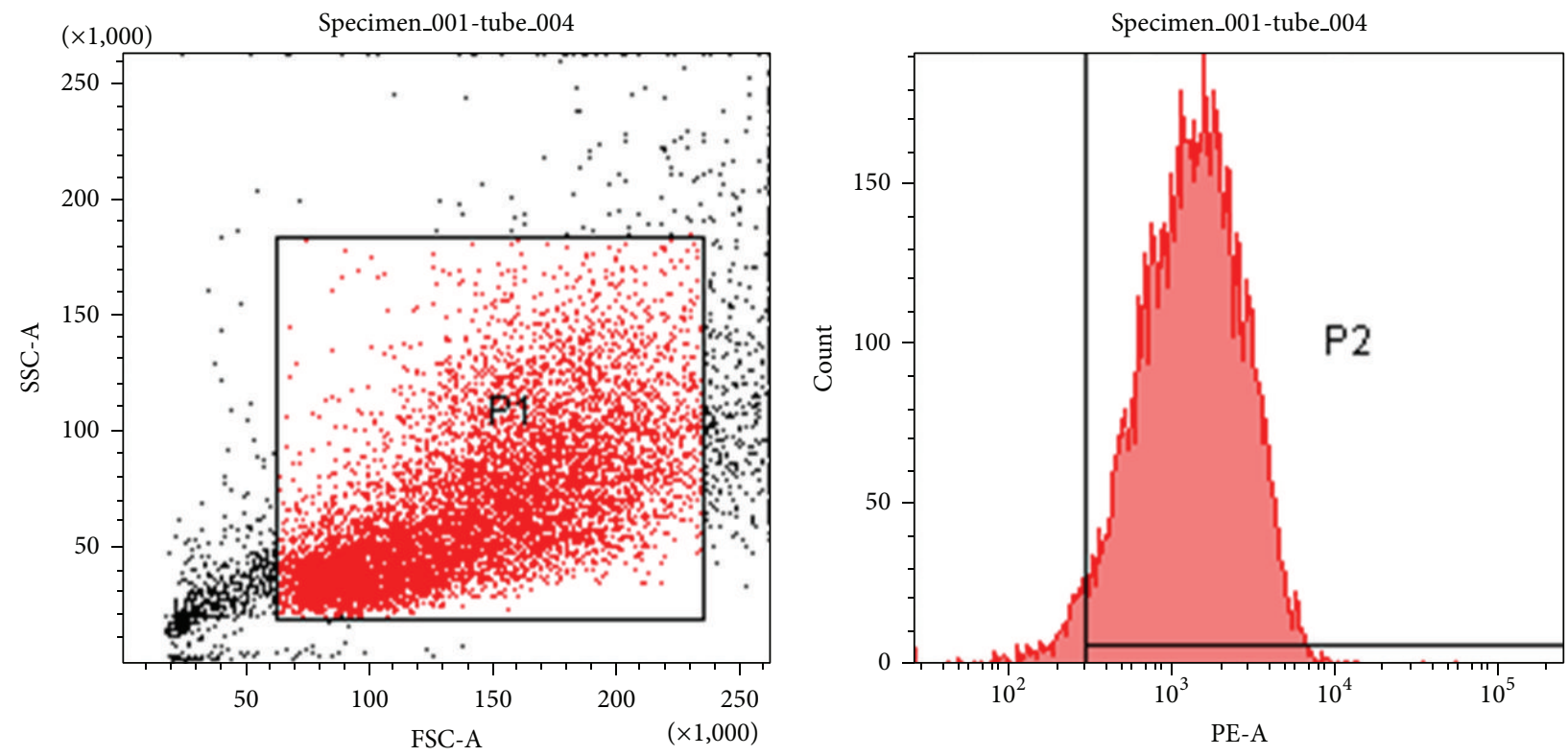

(a)
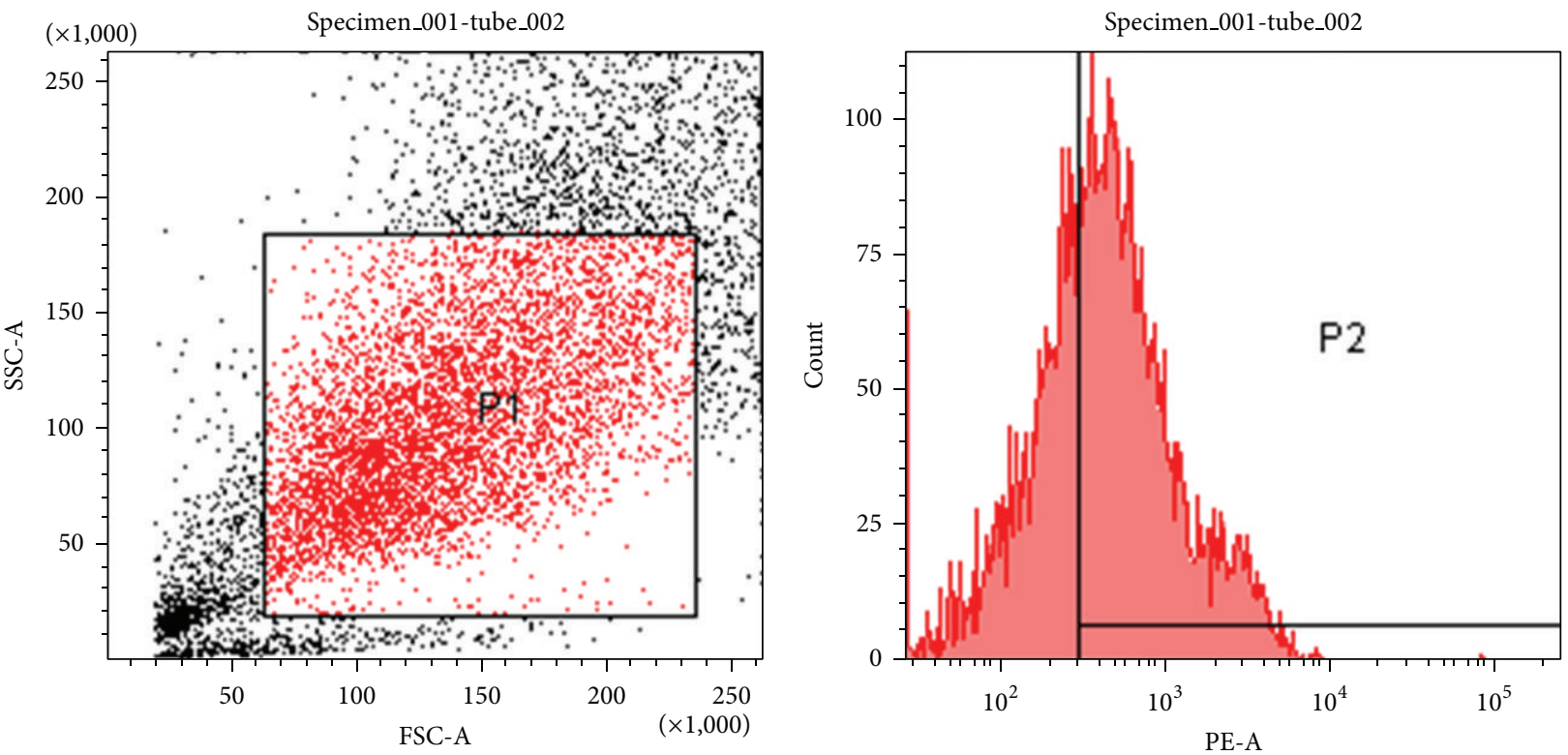

(b)

FIGURE 9: CXCR4 expression on the cell surface after transfection with empty vector (a) or target gene CXCL12-KDEL (b).

the transfection efficiency met the basic outcomes of the study; however, the specific transfection efficiency needs to be clarified by further screening. Meanwhile, application of this method in in vitro experiments can reduce cell surface expression of CXCR4 and the chemotaxis of tumor cells. As such, our results lay a foundation for further screening and animal experiments.

\section{Conclusions}

High expression of CXCR4 may help squamous cancer cells invade local tissues and metastasize to other tissues, and the intracellular sequestration of CXCR4 by transfection of the CXCL12-KDEL fusion gene may inhibit chemotaxis and metastasis in tongue squamous cancer cells.

\section{Conflict of Interests}

The authors declare that there is no conflict of interests regarding the publication of this paper.

\section{References}

[1] C. T. Liao, J. T. Chang, and H. M. Wang, "Analysis of risk factors of predictive local tumor control in oral cavity cancer," Annals of Surgical Oncology, vol. 15, no. 3, pp. 915-922, 2008. 
[2] L. P. Kowalski, R. Bagietto, J. R. Lara, and et al, "Prognostic significance of the distribution of neck node metastasis from oral carcinoma," Head \& Neck, vol. 22, no. 3, pp. 207-214, 2000.

[3] M. Noguchi, Y. Kido, H. Kubota, H. Kinjo, and G. Kohama, "Prognostic factors and relative risk for survival in N1-3 oral squamous cell carcinoma: a multivariate analysis using Cox's hazard model," British Journal of Oral and Maxillofacial Surgery, vol. 37, no. 6, pp. 433-437, 1999.

[4] J. A. Woolgar, S. Rogers, C. R. West, R. D. Errington, J. S. Brown, and E. D. Vaughan, "Survival and patterns of recurrence in 200 oral cancer patients treated by radical surgery and neck dissection," Oral Oncology, vol. 35, no. 3, pp. 257-265, 1999.

[5] M. P. Crump, J. H. Gong, P. Loetscher et al., "Solution structure and basis for functional activity of stromal cell-derived factor-1: dissociation of CXCR4 activation from binding and inhibition of HIV-1," EMBO Journal, vol. 16, no. 23, pp. 6996-7007, 1997.

[6] H. Kang, G. Watkims, A. Douglas-Jones, and et al, “The elevated level of CXCR4 is correlated with nodal metastasis of human breast cancer," The Breast, vol. 14, no. 5, pp. 360-367, 2005.

[7] J. J. Liang, S. Zhu, R. Bruggeman et al., "High levels of expression of human stromal cell-derived factor-1 are associated with worse prognosis in patients with stage II pancreatic ductal adenocarcinoma," Cancer Epidemiology, Biomarkers \& Prevention, vol. 19, no. 10, pp. 2598-2604, 2010.

[8] M. Darash-Yahana, E. Pikarsky, R. Abramovitch et al., "Role of high expression levels of CXCR4 in tumor growth, vascularization, and metastasis," The FASEB Journal, vol. 18, no. 11, pp. 1240-1242, 2004.

[9] A. Almofti, D. Uchida, N. M. Begum et al., "The clinicopathological significance of the expression of CXCR4 protein in oral squamous cell carcinoma," International Journal of Oncology, vol. 25, no. 1, pp. 65-71, 2004.

[10] J. Libura, J. Drukala, M. Majka, and etal, "CXCR4-SDF-1 signaling is active in rhabdomyosarcoma cells and regulates locomotion, chemotaxis, and adhesion," Blood, vol. 100, no. 7, pp. 2597-2606, 2002.

[11] T. Kijima, G. Maulik, P. C. Ma et al., "Regulation of cellular proliferation, cytoskeletal function, and signal transduction through CXCR4 and c-Kit in small cell lung cancer cells," Cancer Research, vol. 62, no. 21, pp. 6304-6311, 2002.

[12] G. Lazennec and A. Richmond, "Chemokines and chemokine receptors: new insights into cancer-related inflammation," Trends in Molecular Medicine, vol. 16, no. 3, pp. 133-144, 2010.

[13] A. Orimo, P. B. Gupta, D. C. Sgroi et al., "Stromal fibroblasts present in invasive human breast carcinomas promote tumor growth and angiogenesis through elevated SDF-1/CXCL12 secretion," Cell, vol. 121, no. 3, pp. 335-348, 2005.

[14] R. M. Strieter, M. D. Burdick, J. Mestas, B. Gomperts, M. P. Keane, and J. A. Belperio, "Cancer CXC chemokine networks and tumour angiogenesis," European Journal of Cancer, vol. 42, no. 6, pp. 768-778, 2006.

[15] M. Kucia, K. Jankowski, R. Reca et al., "CXCR4-SDF-1 signalling, locomotion, chemotaxis and adhesion," Journal of Molecular Histology, vol. 35, no. 3, pp. 233-245, 2004.

[16] Y.-R. Zou, A. H. Kottman, M. Kuroda, I. Taniuchi, and D. R. Littman, "Function of the chemokine receptor CXCR4 in heaematopolesis and in cerebellar development," Nature, vol. 393, no. 6685, pp. 595-599, 1998.

[17] A. Aiuti, I. J. Webb, C. Bleul, T. Springer, and J. C. GutierrezRamos, "The chemokine SDF-1 is a chemoattractant for human $\mathrm{CD} 34+$ hematopoietic progenitor cells and provides a new mechanism to explain the mobilization of CD34+ progenitors to peripheral blood," Journal of Experimental Medicine, vol. 185, no. 1, pp. 111-120, 1997.

[18] Q. Ma, D. Jones, P. R. Borghesani et al., "Impaired Blymphopoiesis, myelopoiesis, and derailed cerebellar neuron migration in CXCR4- and SDF-1-deficient mice," Proceedings of the National Academy of Sciences of the United States of America, vol. 95, no. 16, pp. 9448-9453, 1998.

[19] A. Zlotnik, "New insights on the role of CXCR4 in cancer metastasis," Journal of Pathology, vol. 215, no. 3, pp. 211-213, 2008.

[20] F. Balkwill, "The significance of cancer cell expression of the chemokine receptor CXCR4," Seminars in Cancer Biology, vol. 14, no. 3, pp. 171-179, 2004.

[21] B. Furusato, A. Mohamed, M. Uhlén, and J. S. Rhim, "CXCR4 and cancer: review Article," Pathology International, vol. 60, no. 7, pp. 497-505, 2010.

[22] J. Vandercappellen, J. van Damme, and S. Struyf, "The role of CXC chemokines and their receptors in cancer," Cancer Letters, vol. 267, no. 2, pp. 226-244, 2008.

[23] M. Darash-Yahana, E. Pikarsky, R. Abramovitch et al., "Role of high expression levels of CXCR4 in tumor growth, vascularization, and metastasis," FASEB Journal, vol. 18, no. 11, pp. 12401242, 2004.

[24] R. K. Ganju, S. A. Brubaker, J. Meyer et al., "The $\alpha$-chemokine, stromal cell-derived factor- $1 \alpha$, binds to the transmembrane Gprotein-coupled CXCR- 4 receptor and activates multiple signal transduction pathways," The Journal of Biological Chemistry, vol. 273, no. 36, pp. 23169-23175, 1998.

[25] A. Muller, B. Homey, H. Soto et al., "Involvementof chemokine receptors in breast cancermetastasi," Nature, vol. 410, pp. 50-56, 2001.

[26] L. Wang, Z. Wang, X. Liu, and et al, "High-level C-X-C chemokine receptor type 4 expression correlates with brainspecific metastasis following complete resection of non-small cell lung cancer," Oncology Letters, vol. 7, no. 6, pp. 1871-1876, 2014.

[27] J. Kim, H. Takeuchi, S. T. Lam et al., "Chemokine receptor CXCR4 expression in colorectal cancer patients increases the risk for recurrence and for poor survival," Journal of Clinical Oncology, vol. 23, no. 12, pp. 2744-2753, 2005.

[28] S. Saigusa, Y. Toiyama, K. Tanaka et al., "Stromal CXCR4 and CXCL12 expression is associated with distant recurrence and poor prognosis in rectal cancer after chemoradiotherapy," Annals of Surgical Oncology, vol. 17, no. 8, pp. 2051-2058, 2010.

[29] K. Koishi, R. Yoshikawa, T. Tsujimura et al., "Persistent CXCR4 expression after preoperative chemoradiotherapy predicts early recurrence and poor prognosis in esophageal cancer," World Journal of Gastroenterology, vol. 12, no. 47, pp. 7585-7590, 2006.

[30] W. C. Liao, H. P. Wang, H. Y. Huang et al., "CXCR4 expression predictsearly liver recurrence and poor survival after resection of pancreatic adenocarcinoma," Clinical and Translational Gastroenterology, vol. 3, article e22, 2012.

[31] L. Wang, W. Chen, L. Gao et al., "High expression of CXCR4, CXCR7 and SDF-1 predicts poor survival in renal cell carcinoma," World Journal of Surgical Oncology, vol. 10, article 212, 2012.

[32] A. S. Felix, R. A. Stone, M. Chivukula et al., "Survival outcomes in endometrial cancer patients are associated with CXCL12 and estrogen receptor expression," International Journal of Cancer, vol. 131, no. 2, pp. E114-E121, 2012. 
[33] B. Guleng, K. Taleishi, M. Ohta, and et al, "Blockade of the stromal cell-derived factor-1/CXCR4 axis attenuates in vivo tumor groeth by inhibiting angiogenesis in a vascular endothelial groeth factor-independent manner," Cancer Research, vol. 65, no. 13, pp. 58-64, 2005.

[34] H. Tamamura, A. Hori, N. Kanzaki et al., "T140 analogs as CXCR4 antagonists identified as anti-metastatic agents in the treatment of breast cancer," FEBS Letters, vol. 550, no. 1-3, pp. 79-83, 2003.

[35] Z. Liang, Y. Yoon, J. Votaw et al., "Silencing of CXCR4 blocks breast cancer metastasis," Cancer Research, vol. 65, no. 3, pp. 967-971, 2005.

[36] I. S. Zeelenberg, L. Ruuls-Van Stalle, and E. Roos, "Retention of CXCR4 in the endoplasmic reticulum blocks dissemination of a T cell hybridoma," Journal of Clinical Investigation, vol. 108, no. 2, pp. 269-277, 2001.

[37] B. Dalby, S. Cates, A. Harris et al., "Advanced transfection with Lipofectamine 2000 reagent: primary neurons, siRNA, and high-throughput applications," Methods, vol. 33, no. 2, pp. 95103, 2004.

[38] J. Kodama, Hasengaowa, T. Kusumoto, and et al, "Association receptor expression and lymph node metastasis in human cervical cancer," Annals of Oncology, vol. 18, no. 1, pp. 70-76, 2007. 


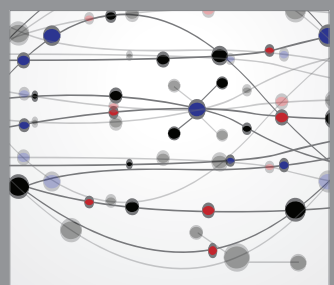

The Scientific World Journal
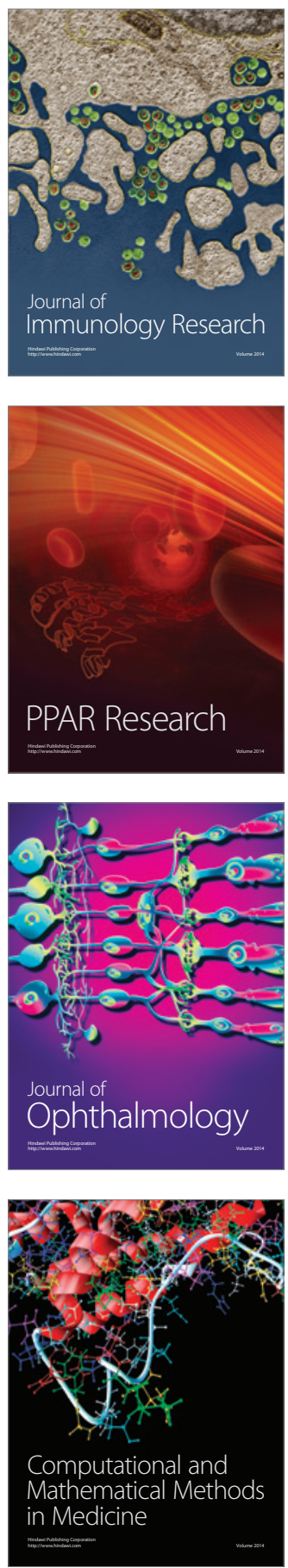

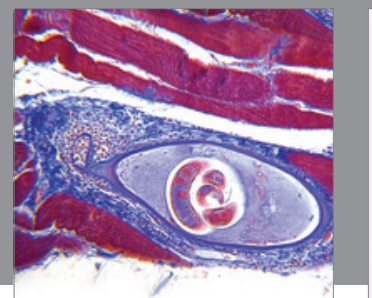

Gastroenterology

Research and Practice
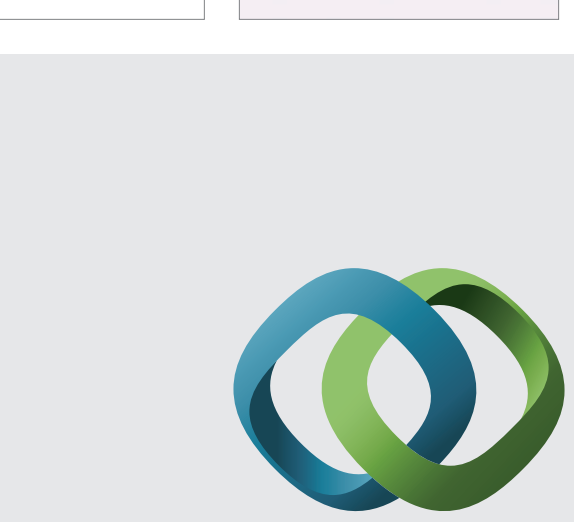

\section{Hindawi}

Submit your manuscripts at

http://www.hindawi.com
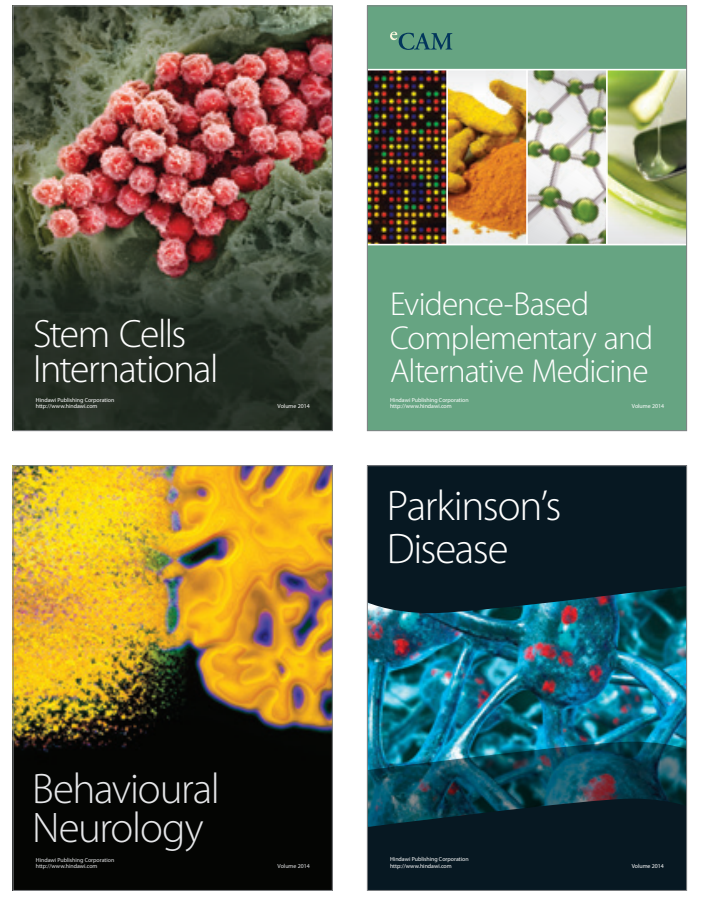
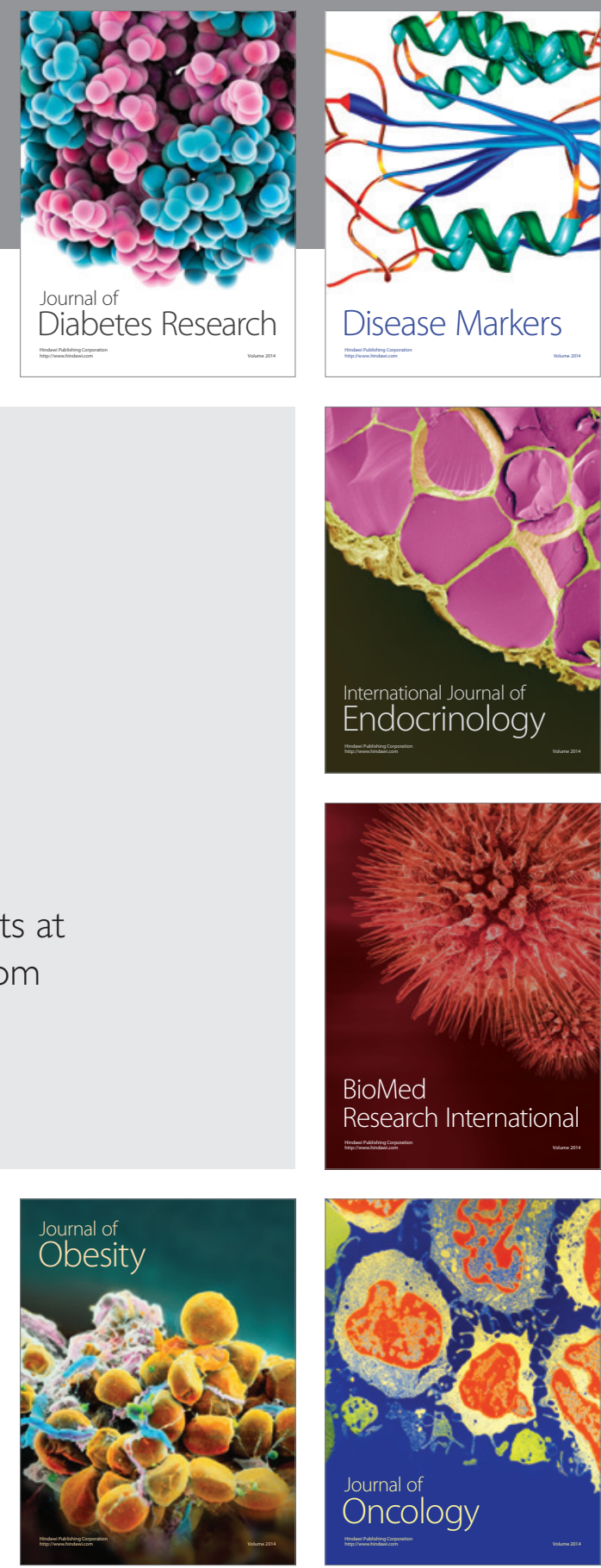

Disease Markers
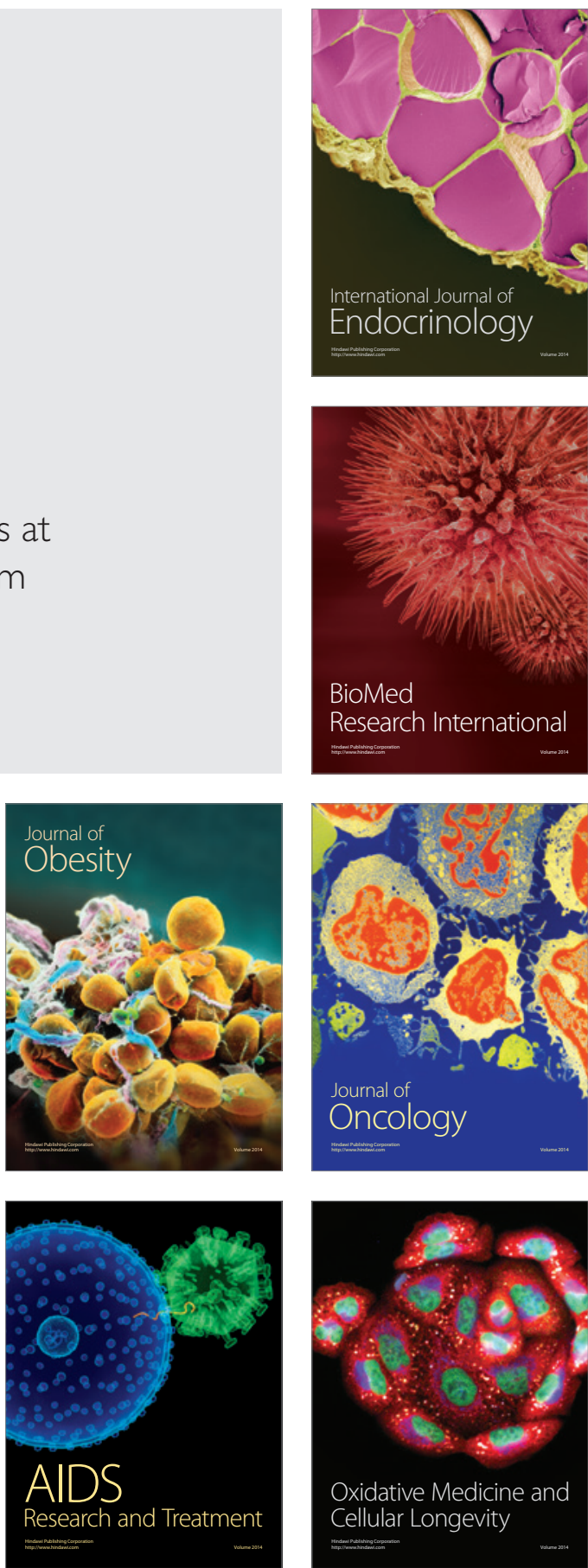